\title{
Energy $\beta$-Conformal Change and Special Finsler Spaces
}

\author{
Amr Soleiman ${ }^{1}$, Amira A. Ishan ${ }^{2}$ \\ ${ }^{1}$ Department of Mathematics, Faculty of Science, Benha University, Benha, Egypt \\ ${ }^{2}$ Department of Mathematics, Taif University, Makkah, KSA \\ Email: amrsoleiman@yahoo.com, amiraishan@hotmail.com
}

Received February 18, 2013; revised March 21, 2013; accepted April 20, 2013

Copyright (C) 2013 Amr Soleiman, Amira A. Ishan. This is an open access article distributed under the Creative Commons Attribution License, which permits unrestricted use, distribution, and reproduction in any medium, provided the original work is properly cited.

\begin{abstract}
The main aim of the present paper is to establish an intrinsic investigation of the energy $\beta$-conformal change of the most important special Finsler spaces, namely, $\mathrm{C}^{\mathrm{h}}$-recurrent, $\mathrm{C}^{\mathrm{v}}$-recurrent, $\mathrm{C}^{0}$-recurrent, $\mathrm{S}^{\mathrm{v}}$-recurrent, quasi-C-reducible, semi-C-reducible, C-reducible, P-reducible, $\mathrm{C}_{2}$-like, $\mathrm{S}_{3}$-like, $\mathrm{P}_{2}$-like and h-isotropic, $\cdots$, etc. Necessary and sufficient conditions for such special Finsler manifolds to be invariant under an energy $\beta$-conformal change are obtained. It should be pointed out that the present work is formulated in a prospective modern coordinate-free form.
\end{abstract}

Keywords: Energy $\beta$-Conformal Change; $C^{\mathrm{h}}$-Recurrent; $\mathrm{C}^{\mathrm{v}}$-Recurrent; $\mathrm{C}^{0}$-Recurrent; $\mathrm{C}_{2}$-Like; Quasi-C-Reducible; C-Reducible; Berwald Space; $\mathrm{S}^{\mathrm{v}}$-Recurrent; $\mathrm{S}_{3}$-Like; $\mathrm{P}_{2}$-Like; h-Isotropic

\section{Introduction}

An important aim of Finsler geometry is the construction of a natural geometric framework of variational calculus and the creation of geometric models that are appropriate for dealing with different physical theories, such as general relativity, relativistic optics, particle physics and others. As opposed to Riemannian geometry, the extra degrees of freedom offered by Finsler geometry, due to the dependence of its geometric objects on the directional arguments, make this geometry potentially more suitable for dealing with such physical theories at a deeper level.

Studying Finsler geometry, however, one encounters substantial difficulties trying to seek analogues of classical global, or sometimes even local, results of Riemannian geometry. These difficulties arise mainly from the fact that in Finsler geometry all geometric objects depend not only on positional coordinates, as in Riemannian geometry, but also on directional arguments.

In Riemannian geometry, there is a canonical linear connection on the manifold $M$, whereas in Finsler geometry there is a corresponding canonical linear connection due to E. Cartan. However, this is not a connection on $\mathrm{M}$ but is a connection on $T(T M)$, the tangent bundle of $T M$, or on $\pi^{-1}(T M)$, the pullback of the tangent bundle $T M$ by $\pi: T M \rightarrow M$. The infinitesimal transformations (changes) in Riemannian and Finsler geometry are important, not only in differential geometry, but also in application to other branches of science, especially in the process of geometrization of physical theories [1].

In [2], we investigated intrinsically energy $\beta$-conformal change of the fundamental linear connections on the pullback bundle of a Finsler manifold, namely, the Cartan connection, the Berwald connection, the Chern connection and the Hashiguchi connection. Moreover, the change of their curvature tensors is obtained.

The present paper is a continuation of [2] where we present an intrinsic investigation of energy $\beta$-conformal change of the most important special Finsler spaces, namely, $\mathrm{C}^{\mathrm{h}}$-recurrent, $\mathrm{C}^{\mathrm{v}}$-recurrent, $\mathrm{C}^{0}$-recurrent, $\mathrm{S}^{\mathrm{v}}$-recurrent, quasi-C-reducible, semi-C-reducible.

C-reducible, P-reducible, $\mathrm{C}_{2}$-like, $\mathrm{S}_{3}$-like, $\mathrm{P}_{2}$-like and h-isotropic, $\cdots$, etc. Moreover, we obtain necessary and sufficient conditions for such special Finsler manifolds to be invariant under an energy $\beta$-conformal change.

Finally, it should be pointed out that all results obtained are formulated in a prospective modern coordinate-free form.

\section{Notation and Preliminaries}

In this section, we give a brief account of the basic concepts of the pullback approach to intrinsic Finsler geometry necessary for this work. For more details, we refer to [3-6].

We assume, unless otherwise stated, that all geometric objects treated are of class $\mathrm{C}^{\infty}$. The following notation will be used throughout this paper:

$M$ : a real paracompact differentiable manifold of finite 
dimension $\mathrm{n}$ and of class $\mathrm{C}^{\infty}$,

$\mathcal{F}(M)$ : the R-algebra of differentiable functions on $M$, $\varkappa(M)$ : the $\mathcal{F}(M)$ : module of vector fields on $M$,

$\pi_{M}: T M \rightarrow M:$ the tangent bundle of $M$,

$\pi_{M}^{*}: T^{*} M \rightarrow M:$ the cotangent bundle of $M$,

$\pi: \mathcal{T M} \rightarrow M:$ the subbundle of nonzero vectors tan-

gent to $M$,

$V(T M)$ : the vertical subbundle of the bundle TTM,

$P: \pi^{-1}(T M) \rightarrow T M:$ the pullback of the tangent bundle $T M$ by $\pi$,

$P^{*}: \pi^{-1}\left(T^{*} M\right) \rightarrow T M:$ the pullback of the cotangent bundle $T * M$ by $\pi$,

$\chi(\pi(M)):$ the $\mathcal{F}(T M)$ : module of differentiable sections of $\pi^{-1}(T M)$,

$\chi^{*}(\pi(M))$ : the $\mathcal{F}(T M)$ : module of differentiable sections of $\pi^{-1}(T * M)$,

$i_{X}$ : the interior product with respect to $X \in \varkappa(M)$,

df: the exterior derivative of $f \in \mathcal{F}(M)$,

$\mathrm{d} L:=\left[i_{L}, d\right], \mathrm{i}_{\mathrm{L}}$ being the interior derivative with respect to a vector form $L$.

Elements of $\varkappa(\pi(M))$ will be called $\pi$-vector fields and will be denoted by barred letters. Tensor fields on $\pi^{-1}(T M)$ will be called $\pi$-tensor fields. The fundamental $\pi$-vector field is the $\pi$-vector field $\bar{\eta}$ defined by $\bar{\eta}(u)=(u, u)$ for all $\mathrm{u} \in \mathcal{T} M$.

We have the following short exact sequence of vector bundles, relating the tangent bundle $T(T M)$ and the pullback bundle $\pi^{-1}(T M)$ :

$$
0 \rightarrow \pi^{-1}(M) \stackrel{\gamma}{\longrightarrow} T(T M) \stackrel{\rho}{\longrightarrow} \pi^{-1}(T M) \rightarrow 0,
$$

where the bundle morphisms $\rho$ and $\gamma$ are defined respectively by $\rho:=\left(\pi_{T M}, \mathrm{~d} \pi\right)$ and $\gamma(u, v):=j_{u}(v)$, where $\mathrm{j}_{\mathrm{u}}$ is the natural isomorphism

$j_{u}: T \pi_{M(v)} M \rightarrow T_{u}\left(T \pi_{M(v)} M\right)$.

The vector 1-form $J$ on $T M$ defined by $J:=\gamma \circ \rho$ is called the natural almost tangent structure of $T M$. The vertical vector field $C$ on $T M$ defined by $C:=\gamma \circ \bar{\eta}$ is called the fundamental or the canonical (Liouville) vector field.

Let $D$ be a linear connection (or simply a connection) on the pullback bundle $\pi^{-1}(T M)$. We associate with $D$ the map

$$
K: T M \rightarrow \pi^{-1}(T M): X \mapsto D_{X} \bar{\eta}
$$

called the connection (or the deflection) map of $D$. A tangent vector $X \in T_{u}(T M)$ is said to be horizontal if $K(X)=0$. The vector space

$H_{u}(T M)=\left\{X \in T_{u}(T M): K(X)=0\right\}$ of the horizontal vectors at $u \in T M$ is called the horizontal space to $M$ at $u$. The connection $\mathrm{D}$ is said to be regular if

$$
T_{u}(T M)=V_{u}(T M) \oplus H_{u}(T M) \forall u \in T M .
$$

If $M$ is endowed with a regular connection, then the vector bundle maps

$$
\begin{aligned}
& \gamma: \pi^{-1}(T M) \rightarrow V(T M), \\
& \left.\rho\right|_{H(T M)}: H(T M) \rightarrow \pi^{-1}(T M), \\
& \left.K\right|_{V(T M)}: V(T M) \rightarrow \pi^{-1}(T M) .
\end{aligned}
$$

are vector bundle isomorphisms. Let us denote $\beta:=\left(\left.\rho\right|_{H(T M)}\right)^{-1}$, this map will be called the horizontal map of the connection $D$. According to the direct sum decomposition (1), a regular connection $D$ gives rise to a horizontal projector $h_{D}$ and a vertical projector $v_{D}$, given by

$$
h_{D}=\beta \circ \rho, v_{D}=I-\beta \circ \rho,
$$

where I is the identity endomorphism on $T(T M)$.

The (classical) torsion tensor $T$ of the connection $D$ is defined by

$$
\begin{aligned}
& \boldsymbol{T}(X, Y)=D_{X} \rho Y-D_{Y} \rho X-\rho[X, Y], \\
& \forall X, Y \in \varkappa(T M) .
\end{aligned}
$$

The horizontal ((h)h-) and mixed ((h)hv-) torsion tensors, denoted by $Q$ and T respectively, are defined by

$$
\begin{aligned}
& Q(\bar{X}, \bar{Y})=T(\beta \bar{X}, \beta \bar{Y}), T(\bar{X}, \bar{Y})=T(\gamma \bar{X}, \beta \bar{X}), \\
& \forall \bar{X}, \bar{Y} \in \chi(\pi(M)) .
\end{aligned}
$$

The (classical) curvature tensor $\mathrm{K}$ of the connection $\mathrm{D}$ is defined by

$$
\begin{aligned}
& K(X, Y) \rho Z=-D_{X} D_{Y} \rho Z+D_{Y} D_{X} \rho Z+D_{[X, Y]} \rho Z, \\
& \forall X, Y, Z \in \chi(T M) .
\end{aligned}
$$

The horizontal (h-), mixed (hv-) and vertical (v-) curvature tensors, denoted by $R, P$ and $S$ respectively, are defined by

$$
\begin{aligned}
& R(\bar{X}, \bar{Y}) \bar{Z}=K(\beta \bar{X}, \beta \bar{Y}) \bar{Z}, \\
& P(\bar{X}, \bar{Y}) \bar{Z}=K(\beta \bar{X}, \gamma \bar{Y}) \bar{Z}, \\
& S(\bar{X}, \bar{Y}) \bar{Z}=K(\gamma \bar{X}, \gamma \bar{Y}) \bar{Z} .
\end{aligned}
$$

The contracted curvature tensors, denoted by , $\hat{R}, \hat{P}$ and $\hat{S}$ respectively, are also known as the (v)h-, (v)hvand (v)v-torsion tensors and are defined by

$$
\begin{aligned}
& \hat{R}(\bar{X}, \bar{Y})=R(\bar{X}, \bar{Y}) \bar{\eta}, \\
& \hat{P}(\bar{X}, \bar{Y})=P(\bar{X}, \bar{Y}) \bar{\eta}, \\
& \hat{S}(\bar{X}, \bar{Y})=S(\bar{X}, \bar{Y}) \bar{\eta} .
\end{aligned}
$$

If $M$ is endowed with a metric $g$ on $\pi^{-1}(T M)$, we write

$$
R(\bar{X}, \bar{Y}, \bar{Z}, \bar{W}):=g(R(\bar{X}, \bar{Y}) \bar{Z}, \bar{W})
$$

On a Finsler manifold $(M, L)$, there are canonically 
associated four linear connections on $\pi^{-1}(T M)$ [7]: the Cartan connection $\nabla$, the Chern (Rund) connection $D^{c}$, the Hashiguchi connection $D^{*}$ and the Berwald connection $\mathrm{D}^{\circ}$. Each of these connections is regular with (h) hv-torsion $T$ satisfying $T(\bar{X}, \bar{\eta})=0$. The following theorem guarantees the existence and uniqueness of the Cartan connection on the pullback bundle.

Theorem 2.1. [8] Let $(M, L)$ be a Finsler manifold and $g$ the Finsler metric defined by $L$. There exists a unique regular connection $\nabla$ on $\pi^{-1}(T M)$ such that

(a) $\nabla$ is metric: $\nabla g=0$,

(b) The (h) h-torsion of $\nabla$ vanishes: $Q=0$,

(c) The (h) hv-torsion $\mathrm{T}$ of $\nabla$ satisfies:

$$
g(T(\bar{X}, \bar{Y}), \bar{Z})=g(T(\bar{X}, \bar{Z}), \bar{Y})
$$

Definition 2.2. Let $(M, L)$ be a Finsler manifold and $\mathrm{g}$ the Finsler metric dened by $L$. We define:

$$
\ell(X):=L^{-1} g(\bar{X}, \bar{\eta}),
$$

$\hbar:=g-\ell \otimes \ell:$ the angular metric tensor,

$T(\bar{X}, \bar{Y}, \bar{Z}):=g(T(\bar{X}, \bar{Y}), \bar{Z}):$ the Cartan tensor,

$C(\bar{X}):=\operatorname{Tr}\{\bar{Y} \rightarrow T(\bar{X}, \bar{Y})\}:$ the contracted torsion,

$g(\bar{C}, \bar{X}):=C(X): \bar{C}$ is the $\pi$-vector field associated with the $\pi$-form $C$, tensor,

$\operatorname{Ric}^{v}(\bar{X}, \bar{Y}):=\operatorname{Tr}\{\bar{Z} \rightarrow S(\bar{X}, \bar{Z}) \bar{Y}\}:$ the vertical Ricci

$$
g\left(\operatorname{Ric}_{0}^{v}(\bar{X}), Y\right):=\operatorname{Ric}^{v}(\bar{X}, \bar{Y}): \text { the vertical Ricci map, }
$$
ture.

$S c^{v}:=\operatorname{Tr}\left\{\bar{X} \rightarrow \operatorname{Ric}_{0}^{v}(\bar{X})\right\}:$ the vertical scalar curva-

Deicke theorem [9] can be formulated globally as follows:

Lemma 2.3. Let $(M, L)$ be a Finsler manifold. The following assertions are equivalent:

(a) $(M, L)$ is Riemannian,

(b) The (h)hv-torsion tensor $T$ vanishes,

(c) The $\pi$-form $C$ vanishes.

Concerning the Berwald connection on the pullback bundle, we have

Theorem 2.4. [8] Let $(M, L)$ be a Finsler manifold. There exists a unique regular connection $\mathrm{D}^{\circ}$ on $\pi^{-1}(T M)$ such that

(a) $\mathrm{D}_{\mathrm{h} \circ \mathrm{X}}^{\circ} \mathrm{L}=0$,

(b) $\mathrm{D}^{\circ}$ is torsion-free: $T=0$,

(c) The (v)hv-torsion $\mathrm{P}^{\circ}$ of $\mathrm{D}^{\circ}$ vanishes $: \hat{\mathrm{P}}^{\circ}(\overline{\mathrm{X}}, \overline{\mathrm{Y}})=0$.

Such a connection is called the Berwald connection associated with the Finsler manifold $(M, L)$.

We terminate this section by some concepts and results concerning the Klein-Grifone approach to intrinsic Finsler geometry. For more details, we refer to [10-13].

A semispray is a vector field $\mathrm{X}$ on $\mathrm{TM}, \mathrm{C}^{\infty}$ on $\mathcal{T} \mathrm{M}, \mathrm{C}^{1}$ on TM, such that $\rho \circ X=\bar{\eta}$.
A semispray $\mathrm{X}$ which is homogeneous of degree 2 in the directional argument $([C, X]=X)$ is called a spray.

Proposition 2.5. [12] Let $(M, L)$ be a Finsler manifold. The vector field $\mathrm{G}$ on TM defined by $i_{G} \Omega=-\mathrm{d} E$ is a spray, where $E:=(1 / 2) L^{2}$ is the energy function and $\Omega:=\mathrm{d} d_{J} E$. Such a spray is called the canonical spray.

A nonlinear connection on $M$ is a vector 1 -form $\Gamma$ on $\mathrm{TM}, \mathrm{C}^{\infty}$ on $\mathcal{T} \mathrm{M}, \mathrm{C}^{0}$ on $T M$, such that

$$
J \Gamma=J, \Gamma J=-J .
$$

The horizontal and vertical projectors $\mathrm{h}_{\Gamma}$ and $\mathrm{v}_{\Gamma}$ associated with $\Gamma$ are defined by

$$
h_{\Gamma}:=(1 / 2)(I+\Gamma), v_{\Gamma}:=(1 / 2)(I-\Gamma) .
$$

To each nonlinear connection $\Gamma$ there is associated a semispray $\mathrm{S}$ defined by $S=h_{\Gamma} S^{\prime}$, where $S^{\prime}$ is an arbitrary semispray. A nonlinear connection $\Gamma$ is homogeneous if $[C, \Gamma]=0$. The torsion of a nonlinear connection $\Gamma$ is the vector 2 -form $t$ on $T M$ defined by $t:=(1 / 2)[J, \Gamma]$. The curvature of $\Gamma$ is the vector 2 -form $\mathfrak{R}$ on TM defined by $\mathfrak{R}:=-(1 / 2)\left[h_{\Gamma}, h_{\Gamma}\right]$. A nonlinear connection $\Gamma$ is said to be conservative if $\mathrm{d} h_{\Gamma} E=0$.

Theorem 2.6. [11] On a Finsler manifold $(M, L)$, there exists a unique conservative homogenous nonlinear connection with zero torsion. It is given by:

$$
\Gamma=[J, G],
$$

where $G$ is the canonical spray. Such a nonlinear connection is called the canonical connection, the Barthel connection or the Cartan nonlinear connection associated with $(M, L)$.

It should be noted that the semispray associated with the Barthel connection is a spray, which is the canonical spray.

\section{Energy $\beta$-Conformal Change and Special Finsler Spaces}

In [2], we investigated intrinsically a particular $\beta$-change, called an energy $\beta$-conformal change:

$$
\tilde{L}^{2}(x, y)=\mathrm{e}^{2 \sigma(x)} L^{2}(x, y)+B^{2}(x, y),
$$

where $(M, L)$ is a Finsler manifold admitting a concurrent $\pi$-vector field $\bar{\zeta}, B:=g(\bar{\zeta}, \bar{\eta}) ; \bar{\eta}$ being the fundamental $\pi$-vector field and $\sigma(\mathrm{x})$ is a function on $M$. Moreover, the relation between the two Barthel connections $\Gamma$ and $\tilde{\Gamma}$, corresponding to this change, is obtained. The energy $\beta$-conformal change of the fundamental linear connections on the pullback bundle of a Finsler manifold is studied.

In this section, we introduce the effect of energy $\beta$-conformal change on some important special Finsler spaces. The intrinsic definitions of the special Finsler spaces treated here are quoted from [14]. 
The following definition and three lemmas are useful for subsequence use.

Definition 3.1. [15] Let $(M, L)$ be a Finsler manifold. A $\pi$-vector field $\bar{\zeta} \in(\pi(M))$ is called a concurrent $\pi$-vector field if it satisfies the following conditions

$$
\nabla_{\gamma \bar{X}} \bar{\zeta}=-X, \nabla_{\beta \bar{X}} \bar{\zeta}=0
$$

In other words, $\bar{\zeta}$ is a concurrent $\pi$-vector field if $\nabla_{X} \bar{\zeta}=-\rho X$ for all $X \in \varkappa(T M)$.

Lemma 3.2. [15] Let $\bar{\zeta} \in \varkappa(\pi(M))$ be a concurrent $\pi$-vector field. For every $\bar{X}, \bar{Y} \in \varkappa(\pi(M))$, we have

(a) $T(\bar{X}, \bar{\zeta})=T(\bar{\zeta}, \bar{X})=0$,

(b) $\hat{P}(\bar{X}, \bar{\zeta})=\hat{P}(\bar{\zeta}, \bar{X})=0$,

(c) $P(\bar{X}, \bar{\zeta}) \bar{Y}=P(\bar{\zeta}, \bar{X}) \bar{Y}=0$.

Lemma 3.3. [15] Let $(M, L)$ be a Finsler manifold which admits a concurrent $\pi$-vector $\bar{\zeta}$. Then, we have:

(a) The concurrent $\pi$-vector eld $\bar{\zeta}$ is everywhere non -zero.

(b) The scalar function $B:=g(\bar{\zeta}, \bar{\eta})$ is everywhere non-zero.

(c) The $\pi$-vector field $\bar{m}:=\bar{\zeta}-\left(B / L^{2}\right) \bar{\eta}$ is everywhere non-zero and is orthogonal to $\bar{\eta}$.

(d) The $\pi$-vector fields $\bar{m}$ and $\bar{\zeta}$ satisfy $g(\bar{m}, \bar{\zeta})=g(\bar{m}, \bar{m}) \neq 0$.

(e) The angular metric tensor $\hbar$ satisfies $\hbar(\bar{\zeta}, X) \neq 0$ for all $\bar{X} \neq \bar{\eta}$.

Lemma 3.4. [2] Under the energy $\beta$-conformal change (4), we have

$$
\tilde{h}=h-L, \tilde{v}=v+L,
$$

or equivalently $\tilde{\beta}=\beta-L \beta, \tilde{K}=K+K L$,

where

$$
\begin{gathered}
E:=\left\{E[\gamma \bar{\sigma}, J]-d_{J} E \otimes \gamma \bar{\sigma}+\sigma_{1} J+d \sigma \otimes C\right\} \\
-\left(1 /\left(\mathrm{e}^{2 \sigma(x)}+p^{2}\right)\right)\left\{\left(1-\sigma_{2}\right) d_{J} E+B \mathrm{~d} \sigma+\sigma_{1} d_{J} B\right\} \otimes \gamma \bar{\zeta} ; \\
g(\rho X, \bar{\sigma}):=\mathrm{d} \sigma(X)=\mathrm{d} \sigma(h X), \\
\sigma_{1}:=\mathrm{d} \sigma(G), p^{2}:=g(\bar{\zeta}, \bar{\zeta}) \\
\text { and } \sigma_{2}:=\mathrm{d} \sigma(\beta) .
\end{gathered}
$$

Theorem 3.5. [2] Let $(M, L)$ and $(M, L)$ be two Finsler manifolds related by the energy $\beta$-conformal change (4). Then the associated Cartan connections $\nabla$ and $\tilde{\nabla}$ are related by:

$$
\tilde{\nabla}_{X} \bar{Y}=\nabla_{X} \bar{Y}+\omega(X, \bar{Y})
$$

where

$$
\begin{aligned}
\omega(X, \bar{Y}):= & (\mathrm{d} \sigma(h X)) \bar{Y}+(\mathrm{d} \sigma(\beta \bar{Y})) \rho X-g(\rho X, \bar{Y}) \bar{\sigma} \\
- & T( \pm \beta \bar{Y}, \rho X)+T^{\prime}( \pm X, \beta \bar{Y}) \\
- & \left(1 /\left(\mathrm{e}^{2 \sigma(x)}+p^{2}\right)\right) \\
& \cdot\{\mathrm{d} \sigma(h X) g(\bar{Y}, \bar{\zeta})+\mathrm{d} \sigma(\beta \bar{Y}) g(\rho X, \bar{\zeta}) \\
& -g(\bar{\sigma}, \bar{\zeta}) g(\rho X, \bar{Y})+g(\rho X, \bar{Y})\} \bar{\zeta}
\end{aligned}
$$

where $T^{\prime}$ is a 2-form on $T M$, with values in $\pi^{-1}(T M)$, defined by

$$
g\left(T^{\prime}(L X, h Y), \rho Z\right)=g(T(L Z, h Y), \rho X) .
$$

Moreover,

(a) The (h) hv-torsion $T$ and the $\pi$-form $\mathrm{C}$ are invariant.

(b) $\tilde{S}(\bar{X}, \bar{Y}) \bar{Z}=S(\bar{X}, \bar{Y}) \bar{Z}$.

(c) $\tilde{P}(\bar{X}, \bar{Y}) \bar{Z}=P(\bar{X}, \bar{Y}) \bar{Z}+V(\bar{X}, \bar{Y}) \bar{Z}$,

where $\mathrm{V}$ is the vector $\pi$-form defined by

$$
\begin{aligned}
& V(\bar{X}, \bar{Y}) \bar{Z} \\
& =-S(\bar{X}, \bar{Y}) \bar{Z}+\left(\nabla_{\gamma \bar{Y}} B\right)(\bar{X}, \bar{Z})+B(T(\bar{X}, \bar{Y}), \bar{Z})
\end{aligned}
$$

(d) $\tilde{R}(\bar{X}, \bar{Y}) \bar{Z}=R(\bar{X}, \bar{Y}) \bar{Z}+H(\bar{X}, \bar{Y}) \bar{Z}$,

where $\mathrm{H}$ is the vector $\pi$-form defined by

$$
\begin{aligned}
& H(\bar{X}, \bar{Y}) \bar{Z}=S(N \bar{X}, N \bar{Y}) Z \\
& -U_{\{\bar{X}, \bar{Y}\}}\left\{P(\bar{X}, N \bar{Y}) \bar{Z}+\left(\nabla_{\beta \bar{X}} B\right)(\bar{Y}, \bar{Z}),\right. \\
& \left.-\left(\nabla_{\gamma N \bar{X}} B\right)(\bar{Y}, \bar{Z})+B(\bar{X}, B(\bar{Y}, \bar{Z}))-B(T(\bar{X}, \bar{Y}), \bar{Z})\right\}^{1}
\end{aligned}
$$

where $B(\bar{X}, \bar{Y}):=\omega(\beta \bar{X}, \bar{Y})$ and $L=: \gamma \circ N \circ \rho$ is given by Lemma 3.4 .

Definition 3.6. A Finsler manifold $(M, L)$ is :

(a) a Berwald manifold if the torsion tensor $\mathrm{T}$ is horizontally parallel: $\nabla_{\beta \bar{X}} T=0$.

(b) a Landsberg manifold if $\hat{P}(\bar{X}, \bar{Y})=0$, or equivalently, if $\nabla_{\beta \bar{\eta}} T=0$.

(c) a general Landsberg manifold if the trace of the linear map $\{\bar{Y} \rightarrow \hat{P}(\bar{X}, \bar{Y})\}$ is identically zero for all $X \in \varkappa(\pi(M))$, or equivalently, if $\nabla_{\beta \bar{\eta}} C=0$.

Now, we have

Theorem 3.7. Let $(M, L)$ be a Finsler manifold which admits a concurrent $\pi$-vector field $\bar{\zeta}$. Under the energy $\beta$-conformal change (4), we have:

(a) if $(M, L)$ is Berwald, then $(M, L)$ and $(M, \tilde{L})$ are Riemannian.

(b) if $(M, L)$ is Landsberg, then $(M, L)$ and $(M, \tilde{L})$ are Riemannian.

${ }^{1} U_{\{\bar{X}, \bar{Y}\}}\{A(\bar{X}, \bar{Y})\}:=A(\bar{X}, \bar{Y})-A(\bar{Y}, \bar{X})$ 


\section{Proof.}

(a) If $(M, L)$ is a Berwald manifold, then $\nabla_{\beta \bar{X}} T=0$. (Definition 3.6(a)). Hence $\nabla_{\beta \bar{\eta}} T=\hat{P}=0$ [16]. Consequently, the hv-curvature $\mathrm{P}$ vanishes [15].

Hence, $0=P(\bar{X}, \bar{Y}, \bar{Z}, \bar{\zeta})=T(\bar{X}, \bar{Y}, \bar{Z})$ by Proposition 3.4(d) of [15]. The result follows then from Deicke theorem (Lemma 2.3) and noting that under $\beta$-conformal change the (h)hv-torsion $\mathrm{T}$ is invariant (Theorem 3.5(a)).

(b) The proof is similar to that of (a).

Definition 3.8. A Finsler manifold $(M, L)$ is said to be:

(a) $\mathrm{C}^{\mathrm{h}}$-recurrent if the (h)hv-torsion tensor $\mathrm{T}$ satisfies the condition $\nabla_{\beta \bar{X}} T=\lambda_{o}(X) T$, where $\lambda_{o}$ is a $\pi$-form of order one.

(b) $\mathrm{C}^{\mathrm{v}}$-recurrent if the (h)hv-torsion tensor $\mathrm{T}$ satisfies the condition $\left(\nabla_{\gamma \bar{X}} T\right)(\bar{Y}, \bar{Z})=\lambda_{o}(\bar{X}) T(\bar{Y}, \bar{Z})$.

(c) $\mathrm{C}^{0}$-recurrent if the (h)hv-torsion tensor $\mathrm{T}$ satisfies the condition $\left(D_{\gamma \bar{X}}^{\circ} T\right)(\bar{Y}, \bar{Z})=\lambda_{o}(\bar{X}) T(\bar{Y}, \bar{Z})$.

Theorem 3.9. Under the energy $\beta$-conformal change (4), we have:

(a) if $(M, L)$ is $\mathrm{C}^{\mathrm{h}}$-recurrent, then $(M, L)$ and $(M, \tilde{L})$ are Riemannian.

(b) if $(M, L)$ is $\mathrm{C}^{\mathrm{v}}$-recurrent, then $(M, L)$ and $(M, \tilde{L})$ are Riemannian.

(c) if $(M, L)$ is $\mathrm{C}^{0}$-recurrent, then $(M, L)$ and $(M, \tilde{L})$ are Riemannian.

\section{Proof.}

(a) We have [16]

$$
\begin{aligned}
& P(\bar{X}, \bar{Y}, \bar{Z}, \bar{W}) \\
= & g\left(\left(\nabla_{\beta \bar{Z}} T\right)(\bar{Y}, \bar{X}), \bar{W}\right)-g\left(\left(\nabla_{\beta \bar{W}} T\right)(\bar{Y}, \bar{X}), \bar{Z}\right) \\
& -g(T(\bar{X}, \bar{W}), \hat{P}(\bar{Z}, \bar{Y}))+g(T(\bar{X}, \bar{Z}), \hat{P}(\bar{W}, \bar{Y})) .
\end{aligned}
$$

Setting $\bar{W}=\bar{\zeta}$ in Equation (10), making use of $\hat{P}(\bar{\zeta}, \bar{X})=0=T(\bar{\zeta}, \bar{X})$ (Lemma 3.2), $P(\bar{X}, \bar{Y}, \bar{Z}, \bar{\zeta})=T(\bar{X}, \bar{Y}, \bar{Z})[15]$ and $g\left(\left(\nabla_{\beta \bar{Z}} T\right)(\bar{X}, \bar{Y}), \bar{W}\right)=g\left(\left(\nabla_{\beta \bar{Z}} T\right)(\bar{X}, \bar{W}), \bar{Y}\right)$ (Proposition 3.3 of [16]), we get

$$
\nabla_{\beta} \bar{\zeta} T=0 .
$$

On the other hand, Definition 3.8(a) for $\bar{X}=\bar{\zeta}$, yields

$$
\nabla_{\beta} \bar{\zeta} T=\lambda_{o}(\bar{\zeta}) T
$$

The above two equations and Theorem 3.5(a) imply that $\tilde{T}=T=0$.

Hence, by Lemma 2.3, the result follows.

(b) and (c) follow from Theorem 4.7 of [14], together with Theorem 3.5(a).

Definition 3.10. A Finsler manifold $(M, L)$ is said to be:

(a) quasi-C-reducible if $\operatorname{dim}(M) \geq 3$ and the Cartan tensor $T$ has the from

$$
\begin{aligned}
T(\bar{X}, \bar{Y}, \bar{Z}) & =A(\bar{X}, \bar{Y}) C(\bar{Z})+A(\bar{Y}, \bar{Z}) C(\bar{X}) \\
& +A(\bar{Z}, \bar{X}) C(\bar{Y}),
\end{aligned}
$$

where $A$ is a symmetric $\pi$-tensor field satisfying

$$
A(\bar{X}, \bar{\eta})=0
$$

(b) semi-C-reducible if $\operatorname{dim} M \geq 3$ and the Cartan tensor $T$ has the form

$$
\begin{aligned}
& T(\bar{X}, \bar{Y}, \bar{Z}) \\
= & (\mu /(n+1)) \\
& \cdot\{\hbar(\bar{X}, \bar{Y}) C(\bar{Z})+\hbar(\bar{Y}, \bar{Z}) C(\bar{X})+\hbar(\bar{Z}, \bar{X}) C(\bar{Y})\} \\
& +\left(\tau / C^{2}\right) C(\bar{X}) C(\bar{Y}) C(\bar{Z}),
\end{aligned}
$$

where $C^{2}:=C(\bar{C}) \neq 0, \mu$ and $\tau$ are scalar functions satisfying $\mu+\tau=1$.

(c) C-reducible if $\operatorname{dim} M \geq 3$ and the Cartan tensor $T$ has the form

$$
\begin{aligned}
& T(\bar{X}, \bar{Y}, \bar{Z}) \\
& =(1 /(n+1)) \\
& \cdot\{\hbar(\bar{X}, \bar{Y}) C(\bar{Z})+\hbar(\bar{Y}, \bar{Z}) C(\bar{X})+\hbar(\bar{Z}, \bar{X}) C(\bar{Y})\} .
\end{aligned}
$$

(d) $\mathrm{C}_{2}$-like if $\operatorname{dim} M \geq 2$ and the Cartan tensor $\mathrm{T}$ has the form

$$
T(\bar{X}, \bar{Y}, \bar{Z})=\left(1 / C^{2}\right) C(\bar{X}) C(\bar{Y}) C(\bar{Z}) .
$$

Theorem 3.11. Under the energy $\beta$-conformal change (4), we have:

(a) if $(M, L)$ is quasi-C-reducible provided that $A(\bar{\zeta}, \bar{\zeta}) \neq 0$, then $(M, L)$ and $(M, \tilde{L})$ are Riemannian.

(b) if $(M, L)$ is C-reducible, then $(M, L)$ and $(M, \tilde{L})$ are Riemannian.

(c) if $(M, L)$ is semi-C-reducible, then $(M, L)$ and $(M, \tilde{L})$ are $\mathrm{C}_{2}$-like.

Proof.

(a) If $(M, L)$ is quasi-C-reducible, then the Cartan tensor $T$ satisfies Relation (11). Setting

$\bar{X}=\bar{Y}=\bar{\zeta}$ into Equation (11) and using the fact that

$$
C(\bar{\zeta})=0(\text { as } C(\zeta):=\operatorname{Tr}\{\bar{X} \rightarrow T(\bar{\zeta}, \bar{X})=0\})
$$

and $T(\bar{X}, \bar{Y}, \bar{\zeta})=g(T(\bar{X}, \bar{\zeta}), \bar{Y})=0$, we get

$$
A(\bar{\zeta}, \bar{\zeta}) C(\bar{X})=0 \text {. }
$$

From which together with the given assumption, $A(\bar{\zeta}, \bar{\zeta}) \neq 0$, it follows that the $\pi$-form $\mathrm{C}$ vanishes. Hence, by Lemma 2.3 and Theorem 3.5(a) imply that 
$\tilde{C}=C=0$. Consequently, again by Lemma 2.3, $(M, L)$ and $(M, \tilde{L})$ are Riemannian manifolds.

(b) Follows from the defining property of C-reducibility by setting $\bar{X}=\bar{Y}=\bar{\zeta}$, taking into account Lemma 3.3(e), Lemma 2.3, Theorem 3.5(a) and $C(\bar{\zeta})=0$.

(c) Let $(M, L)$ be semi-C-reducible. Setting

$\bar{X}=\bar{Y}=\bar{\zeta}$ and $\bar{Z}=\bar{C}$ in Equation (12), taking into account Lemma 3.2(a) and $C(\bar{\zeta})=0$, we get

$$
\mu \hbar(\bar{\zeta}, \bar{\zeta}) C(\bar{C})=0
$$

From which, since $\hbar(\bar{\zeta}, \bar{\zeta}) \neq 0$ and $C(\bar{C}) \neq 0$, it follows that $\mu=0$. Consequently, again by Equation (12), we get

$$
T(\bar{X}, \bar{Y}, \bar{Z})=\left(1 / C^{2}\right) C(\bar{X}) C(\bar{Y}) C(\bar{Z}) .
$$

Now, under the energy $\beta$-conformal change (4), from Theorem 3.5(a), Lemma 2.3 and the Relation [2]

$$
\tilde{g}(\bar{X}, \bar{Y})=\mathrm{e}^{2 \sigma(x)} g(\bar{X}, \bar{Y})+g(\bar{X}, \bar{\zeta}) g(\bar{\zeta}, \bar{Y}),
$$

it follows that $\tilde{T}(\bar{X}, \bar{Y}, \bar{Z})=\mathrm{e}^{2 \sigma(x)} T(\bar{X}, \bar{Y}, \bar{Z})$ and $\tilde{C}^{2}=\mathrm{e}^{-2 \sigma(x)} C^{2}$. Hence, from Equation (14), we get

$$
\tilde{T}(\bar{X}, \bar{Y}, \bar{Z})=\left(1 / \tilde{C}^{2}\right) \tilde{C}(\bar{X}) \tilde{C}(\bar{Y}) \tilde{C}(\bar{Z}) .
$$

Therefore, again from Equations (14), (15) and Definition $3.10(\mathrm{~d})$, the result follows.

Definition 3.12. The condition

$$
\begin{aligned}
& T(\bar{X}, \bar{Y}, \bar{Z}, \bar{W}) \\
& :=L\left(\nabla_{\gamma \bar{X}} T\right)(\bar{Y}, \bar{Z}, \bar{W}) \sum_{(\bar{X}, \bar{Y}, \bar{Z}, \bar{W})}\{\ell(\bar{X}) T(\bar{Y}, \bar{Z}, \bar{W})\}=0^{2},
\end{aligned}
$$

will be called the $T$-condition.

The more relaxed condition

$$
\begin{aligned}
& T_{o}(\bar{X}, \bar{Y}):=L\left(\nabla_{\gamma \bar{X}} C\right)(\bar{Y}) \\
& +\sum_{(\bar{X}, \bar{Y}, \bar{Z}, \bar{W})}\{\ell(\bar{X}) C(\bar{Y})\}=0 .
\end{aligned}
$$

will be called the $T_{o}$-condition.

Theorem 3.13. Under the energy $\beta$-conformal change (4), we have:

(a) if $(M, L)$ satisfies the $T$-condition, then $(M, L)$ and $(M, \tilde{L})$ are Riemannian.

(b) if $(M, L)$ satisfies the $T_{o}$-condition, then $(M, L)$ and $(M, \tilde{L})$ are Riemannian.

\section{Proof.}

(a) If $(M, L)$ satisfies the T-condition, then by setting $\bar{W}=\bar{\zeta}$ into Equation (16), taking into account that $T(\bar{X}, \bar{\zeta})=T(\bar{\zeta}, \bar{X})=0$, we get

$$
\sum_{(\bar{X}, \bar{Y}, \bar{Z})}\{A(\bar{X}, \bar{Y}, \bar{Z})\}:=A(\bar{X}, \bar{Y}, \bar{Z})+A(\bar{Y}, \bar{Z}, \bar{X})+A(\bar{Z}, \bar{X}, \bar{Y})
$$

$$
\ell(\bar{\zeta}) T(\bar{X}, \bar{Y}, \bar{Z})=0
$$

This equation, together with $\ell(\bar{\zeta})=(B / L) \neq 0$ (Lemma 3.3(b)) and Theorem 3.5(a), imply that $\tilde{T}=T=0$. Consequently, by Lemma 2.3, $(M, L)$ and $(M, \tilde{L})$ are Riemannian manifolds.

(b) Follows from Equation (17) by setting $\bar{X}=\bar{\zeta}$, taking into account that

$C(\bar{\zeta})=0,\left(\nabla_{\gamma \bar{X}} C\right)(Y)=\left(\nabla_{\gamma \bar{Y}} C\right)(\bar{X}) \quad$ by

$\left.\left(\nabla_{\gamma \bar{X}} T\right)(\bar{Y}, \bar{Z})=\left(\nabla_{\gamma \bar{Y}} T\right)(\bar{X}, \bar{Z})[16]\right)$,

$\ell(\bar{\zeta})=(B / L) \neq 0$, together with Theorem 3.5(a) and Lemma 3.3.

Definition 3.14. A Finsler manifold $(M, L)$ is said to be $S_{3}$-like if $\operatorname{dim}(M) \geq 4$ and the $v$-curvature tensor $S$ has the form:

$$
\begin{aligned}
& S(\bar{X}, \bar{Y}, \bar{Z}, \bar{W}) \\
= & \left(S c^{v} /(n-1)(n-2)\right) \\
& \times\{\hbar(\bar{X}, \bar{Z}) \hbar(\bar{Y}, \bar{W})-\hbar(\bar{X}, \bar{W}) \hbar(\bar{Y}, \bar{Z})\} .
\end{aligned}
$$

Theorem 3.15. Under the energy $\beta$-conformal change (4), if $(M, L)$ is $\mathrm{S}_{3}$-like with $\operatorname{dim} M \geq 4$, then, the $v$ curvature tensors $\mathrm{S}$ and $\tilde{S}$ vanish.

Proof. Setting $\bar{Z}=\bar{\zeta}$ in Equation (18), taking into account the fact that $S(\bar{X}, \bar{Y}) \bar{\zeta}=0$ (Proposition 3.4(a) of [13]) and $\hbar(\bar{X}, \bar{Y})=: g(\phi(\bar{X}), \bar{Y})$, we immediately get

$\left(S c^{v} /(n-1)(n-2)\right)\{\hbar(\bar{X}, \bar{\zeta}) \phi(\bar{Y})-\hbar(\bar{Y}, \bar{\zeta}) \phi(\bar{X})\}=0$.

Taking the trace of the above equation with respect to $\bar{Y}$, noting that $\operatorname{Tr}(\phi)=n-1 \quad$ [14], we have

$$
\left(S c^{v}(n-1)\right) \hbar(\bar{X}, \bar{\zeta})=0
$$

From which, since $\hbar(\bar{X}, \bar{\zeta}) \neq 0$ (Lemma 3.3(e)), the vertical scalar curvature $S c^{v}$ vanishes.

Now, again from Equation (18) together with Theorem 3.5(b), the result follows.

Definition 3.16. A Finsler manifold $(M, L)$, where $\operatorname{dim} M \geq 3$, is said to be:

(a) $\mathrm{P}_{2}$-like if the hv-curvature tensor $\mathrm{P}$ has the form:

$$
\begin{aligned}
& P(\bar{X}, \bar{Y}, \bar{Z}, \bar{W}) \\
& =\omega(\bar{Z}) T(\bar{X}, \bar{Y}, \bar{W})-\omega(\bar{W}) T(\bar{X}, \bar{Y}, \bar{Z}),
\end{aligned}
$$

where $\omega$ is a (1) $\pi$-form (positively homogeneous of degree 0 ).

(b) P-reducible if the $\pi$-tensor field $\hat{P}(\bar{X}, \bar{Y}, \bar{Z}):=g(\hat{P}(\bar{X}, \bar{Y}, \bar{Z}))$ has the form

$$
\begin{aligned}
& \hat{P}(\bar{X}, \bar{Y}, \bar{Z}) \\
& =\delta(\bar{X}) \hbar(\bar{Y}, \bar{Z})+\delta(\bar{Y}) \hbar(\bar{X}, \bar{Z})+\delta(\bar{Z}) \hbar(\bar{X}, \bar{Y}),
\end{aligned}
$$


where $\delta$ is the $\pi$-form defined by

$$
\delta(\bar{X})=(1 /(n+1))\left(\nabla_{\beta \bar{\eta}} C\right)(\bar{X}) .
$$

Theorem 3.17. Under the energy $\beta$-conformal change (4), we have:

(a) if $(M, L)$ is $\mathrm{P}_{2}$-like, provided that $\omega(\bar{\zeta}) \neq-1$, then $(M, L)$ and $(M, \tilde{L})$ are Riemannian.

(b) if $(M, L)$ is P-reducible, provided that $V(\bar{X}, \bar{Y}) \bar{\eta}=0$ (defined by Theorem 3.5(c)), then $(M, L)$ and $(M, \tilde{L})$ are Landsberg.

\section{Proof.}

(a) Setting $\bar{Z}=\bar{\zeta}$ in Equation (19), taking into account Proposition 3.4(a) of [15] and Lemma 3.2, we immediately get

$$
(\omega(\bar{\zeta})+1) T(\bar{X}, \bar{Y})=0 .
$$

This, together with the given assumption and Theorem 3.5(a), follow that $\tilde{T}$ and $T$ vanish. Hence, by Lemma 2.3, $(M, L)$ and $(M, \tilde{L})$ are Riemannian.

(b) If $(M, L)$ is P-reducible, then, by Definition 3.16(b), the (v)hv-torsion $\hat{P}$ satisfy Relation (20). Setting $\bar{X}=\bar{Y}=\bar{\zeta}$ into Equation (20) and taking into account that $\left(\nabla_{\beta \bar{\eta}} C\right)(\bar{\zeta})=0$, we get

$$
\hbar(\bar{\zeta}, \bar{\zeta})\left(\nabla_{\beta \bar{\eta}} C\right)(\bar{Z})=0 \text {. }
$$

From which, noting that $\hbar(\bar{\zeta}, \bar{\zeta}) \neq 0$ (Lemma 3.3(e)), implies that $\nabla_{\beta \bar{\eta}} C=0$. Hence, again, from Definition 3.16(b) and Theorem 3.5(c) (under the given assumption), the (v)hv-torsion tensors $\hat{P}=P=0$. Hence, by Definition 3.6, the result follows.

Definition 3.18. A Finsler manifold $(M, L)$, where $\operatorname{dim} M \geq 3$, is said to be h-isotropic if there exists a scalar $k_{o}$ such that the horizontal curvature tensor $R$ has the form

$$
R(\bar{X}, \bar{Y}) \bar{Z}=k_{o}\{g(\bar{X}, \bar{Z}) \bar{Y}-g(\bar{Y}, \bar{Z}) \bar{X}\} .
$$

Theorem 3.19. Under the energy $\beta$-conformal change (4), we have:

(a) if $(M, L)$ is h-isotropic, provided that $H(\bar{X}, \bar{Y}) \bar{Z}=0$ (defined by Theorem $3.5(\mathrm{~d})$ ), then the h-curvature tensors $R$ and $\tilde{R}$ of the Cartan connection vanish.

(b) if $(M, L)$ is an h-isotropic Berwald manifold, provided that $H(\bar{X}, \bar{Y}) \bar{Z}=0$, then $(M, L)$ and $(M, \tilde{L})$ are Riemannian.

Proof.

(a) From Definition 3.18(a), we have

$$
\begin{aligned}
& R(\bar{X}, \bar{Y}, \bar{Z}, \bar{W}) \\
& =k_{o}\{g(\bar{X}, \bar{Z}) g(\bar{Y}, \bar{W})-g(\bar{Y}, \bar{Z}) g(\bar{X}, \bar{W})\} .
\end{aligned}
$$

Setting $\bar{Z}=\bar{\zeta}$ and $\bar{X}=\bar{m}$ and noting that $R(\bar{X}, \bar{Y}) \bar{\zeta}=0$ (Proposition 3.4(g) of [15]), we have

$$
k_{o}\{g(\bar{m}, \bar{\zeta}) g(\bar{Y}, \bar{W})-g(\bar{Y}, \bar{\zeta}) g(\bar{m}, \bar{W})\}=0
$$

Taking the trace of this equation, we get

$$
k_{o}(n-1) g(\bar{m}, \bar{\zeta})=0 \text {. }
$$

From which, since $g(\bar{m}, \bar{\zeta})=g(\bar{m}, \bar{m}) \neq 0 \quad$ (Lemma 3.3) and $\operatorname{dim} M \geq 3$, the scalar $k_{o}$ vanishes. Now, again, from Equation (21) and Theorem 3.5(c) (under the given assumption), the h-curvature tensors $R$ and $\tilde{R}$ of the Cartan connection vanish

(b) Follows from (a), taking Theorem 3.7 into account.

\section{Concluding Remark}

It should be pointed out that a global formulation of different aspects of Finsler geometry may give more insight into the infrastructure of physical theories and make a better understanding on the essence of such theories without being trapped into the complications of indices. This is one of the motivations of the present work, where all results obtained are formulated in a prospective modern coordinate-free form. Moreover, it should be noted that the outcome of this work is twofold. Firstly, the local expressions of the obtained results, when calculated, coincide with the existing local results. Secondly, new global proofs have been established.

\section{REFERENCES}

[1] T. Mestdag and V. Toth, Report Mathmatics Physics, Vol. 50, 2002, pp. 167-193. doi:10.1016/S0034-4877(02)80053-2

[2] A. Soleiman, International Journal of Geometric Methods in Modern Physic, Vol. 9, 2012, 21 p.

[3] H. Akbar-Zadeh, "Initiation to Global Finsler Geometry," Elsevier, San Diego, 2006.

[4] P. Dazord, "Propriétés Globales des Géodésiques des Espaces de Finsler," Thése d'Etat, Publication Department Mathematics Lyon, 1969.

[5] A. A. Tamim, "General Theory of Finsler Spaces with Applications to Randers Spaces," Ph.D. Thesis, Cairo University, Cairo, 1991.

[6] N. L. Youssef, S. H. Abed and A. Soleiman, Balkan Journal of Geometry and Its Applications, Vol. 15, 2010, pp. 138-150.

[7] N. L. Youssef, S. H. Abed and A. Soleiman, Tensor N. S., Vol. 71, 2009, pp. 187-208.

[8] N. L. Youssef, S. H. Abed and A. Soleiman, Algebras, Groups and Geometries, Vol. 25, 2008, pp. 363-386.

[9] F. Brickell, Proceedings of the American Mathematical Society, Vol. 16, 1965, pp. 90-191.

[10] J. Grifone, Annales de L'Institut Fourier, Grenoble, Vol. 22, 1972, pp. 287-334. doi:10.5802/aif.407

[11] J. Grifone, Annales de L'Institut Fourier, Grenoble, Vol. 22, 1972, pp. 291-338. doi:10.5802/aif.431 
[12] J. Klein and A. Voutier, Annales de L'Institut Fourier, Grenoble, Vol. 18, 1968, pp. 241-260. doi:10.5802/aif.282

[13] N. L. Youssef, Tensor, N. S., Vol. 36, 1982, pp. 275-280.

[14] N. L. Youssef, S. H. Abed and A. Soleiman, Kyoto Journal of Mathematics, Vol. 48, 2008, pp. 857-893.
[15] N. L. Youssef, S. H. Abed and A. Soleiman, International Journal of Geometric Methods in Modern Physics, Vol. 6, 2009, pp. 1003-1031.

[16] N. L. Youssef, S. H. Abed and A. Soleiman, Journal of the Egyptian Mathematical Society, Vol. 18, 2010, pp. 67-90. 